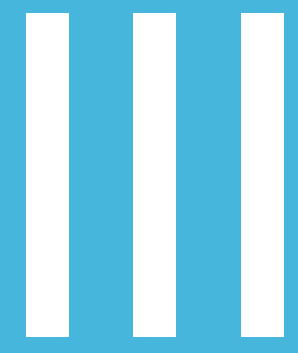

\title{
Autoconcepto y depresión en estudiantes universitarios de una universidad privada
}

Self-concept and depression in undergraduate students at a private university

Universidad Peruana Unión, Perú

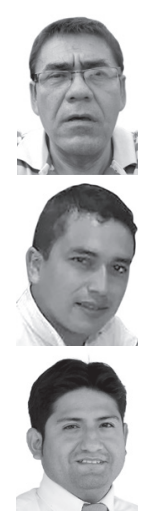

\section{Andrés Fernando Bartra Alegría}

Psicologo por la Universidad Peruana Unión. Licenciado en Educación por la Universidad Nacional Mayor de San Marcos. Investigador y conferenciante. Actualmente se desempeña como docente en centros educativos pertenecientes al Ministerio de Educación del Perú.

\section{Epifanio Felimón, Guerra Sánchez}

Psicólogo por la Universidad Peruana Unión. Investigador y maestrando de psicología educativa.

\section{Renzo Felipe Carranza Esteban}

Psicólogo por la Universidad Peruana Unión. Magister en educación con mención en psicología educativa por la Universidad Peruana Unión. Autor de numerosos artículos de investigación. Ponente internacional y profesor visitante de la Universidad Javeriana de Colombia. Actualmente se desempeña como director de investigación de la Facultad de Ciencias de la Salud de la Universidad Peruana Unión. 
Andrés Fernando Bartra Alegría - Epifanio Felimón, Guerra Sánchez -

Renzo Felipe Carranza Esteban

\section{Resumen}

El objetivo del presente estudio fue determinar la relación que existe entre autoconcepto y depresión en estudiantes universitarios de una Universidad Privada de Tarapoto. Muestra: 465 sujetos, ambos sexos entre 16 y 35 años. Se utilizó un diseño no experimental de tipo descriptivo correlacional. Instrumentos: Escala de Autoconcepto AF5 de García y Musitu y compuesta por 30 ítems y el Inventario de Depresión de Beck Adaptado conformado por 21 ítems. Las propiedades psicométricas de los instrumentos indicaron que son válidos y confiables. Los resultados evidencian que existe una relación inversa altamente significativa entre autoconcepto y depresión en los estudiantes universitarios $\left(r=-.462^{* *}, p<0.01\right)$ lo que indica que a menor autoconcepto mayor depresión.

Palabras clave: Estudiantes, autoconcepto y depresión.

\section{Abstract}

The aim of this study was to assess the relationship between self-concept and depression in undergraduate students of a private university of Tarapoto. The sample consisted of 465 students, both sexes between 16 and 35 years. A non-experimental descriptive correlational design was used. Instruments: AF5 Self-Concept Scale Garcia and Musitu and consists of 30 items and the Beck Depression Inventory Adapted consisting of 21 items. The psychometric properties of the instruments indicated that they are valid and reliable. The results show that there is a highly significant inverse relationship between self-concept and depression in college students ( $r$ $=-.462 * * p<0.01$ ) indicating that major depression to low self-concept.

Keywords: Undergraduate students, self-concept, depression. 


\section{Introducción}

El estudio del hombre, como entender su plenitud, los componentes que forman el carácter y personalidad, ha sido un tema de estudio por parte de los investigadores, sin embargo con el correr de los años -en relación al avance de la ciencia- se ha obtenido distintos resultados. La pregunta que siempre ha cavilado en la mente humana es ¿Quién soy? Ante tal interrogante se fueron entretejiendo varias hipótesis, llegando al punto de elaborar el constructo "autoconcepto", término que fue acuñado el siglo veinte (González y Tourón, 1994). Tal constructo, inicialmente entró en una confusión conceptual, debido a la proliferación de términos usados para definirlos por distintos autores, llegando a la conclusión de definir al autoconcepto: como la percepción de sí mismo, es decir, son todas las actitudes, sentimientos y conocimientos respecto a las propias capacidades, habilidades, apariencia y aceptabilidad social.

El interés por estudiar el autoconcepto deriva de la idea, que las personas que se perciben de manera positiva, miran el mundo de la misma forma, a diferencia de las otras, que se perciben de forma negativa; por tal motivo, al hablar de autoconcepto hacemos énfasis a las conductas del sujeto, pues todas ellas giran en relación al plano personal, social, afectivo e intelectual (Alfaro, 2009).

Es justo en la adolescencia donde se consolida aún más el autoconcepto, por ejemplo: la imagen corporal (una de las dimensiones del autoconcepto), juega un factor importante dentro del proceso de desarrollo. Prácticamente el poseer un autoconcepto adecuado significa que la persona puede generar juicios que le permiten conocerse, reconocerse y definirse en relación de su ser y estar. Pero cuando el adolescente, o la persona misma, no reconocen ni aprecian sus cualidades caen en el famoso estado de "depresión", aquel que empieza con episodios leves y que, en muchas de las veces, puede terminar en grandes tragedias como suicidios entre otros problemas sociales (Vega y Capa, 2008).

Por otro lado, existen pocos estudios en relación a estas dos variables (autoconcepto y depresión) en nuestro país como también en nuestra región; por tal motivo, se elaboró el siguiente estudio de investigación, con la finalidad de descubrir la relación que existe entre las variables ya mencionadas.

\section{Autoconcepto}

El autoconcepto es un constructo que se va formando acorde a las diferentes etapas que atraviesa el ser humano (desde la niñez hasta la edad adulta), el entorno social influye en la estructuralización del autoconcepto (Musito, García y Gutiérrez, 1999).

Revista Apunt. Univ. VOLUMEN VI • NÚMERO 2 p. 53 - 68 
Andrés Fernando Bartra Alegría - Epifanio Felimón, Guerra Sánchez Renzo Felipe Carranza Esteban

La importancia concedida al autoconcepto académico en la investigación educativa es enorme, respondiendo a la presunción de que no se puede entender la conducta escolar sin considerar las percepciones que el sujeto tiene de sí mismo; en particular de su propia competencia académica (Esnaola, 2008). Por otro lado, el autoconcepto social se asocia de manera significativa con numerosos rasgos psicológicos, tales como la estabilidad emocional, la sociabilidad y la responsabilidad. Del mismo modo, las relaciones interpersonales de un individuo son más exitosas cuanto mayor destreza social posee y, como consecuencia se siente mejor consigo mismo, lo que repercute en un aumento de su autoconcepto social (Durá y Garaigordobil, 2005; citado por Esnaola, 2008). Siendo que todos los seres humanos responden ante un estímulo producto de las circunstancias que atraviesa. Al respecto, García, Musitu, Riquelme y Riquelme (2011), explica, que el ser humano ante situaciones favorables sus emociones son gratificadas de manera positiva, lo cual permite crear estímulos adecuados para enfrentar cualquier acontecimiento a venir. Pero ante situaciones no agradables, las emociones son alteradas, impidiendo controlar el estado emocional, acorde a las circunstancias que se va enfrentando.

De otro lado García y Musitu (2001) mencionan que el autoconcepto familiar es el componente principal, que permite a cada persona enfrentar los desafíos del diario vivir. Este componente es desarrollado durante la niñez, donde van formando imágenes modelos de autoconcepto de su entorno familiar. Asimismo, cada niño se encuentra etiquetado invisiblemente, algunos de forma positiva, a través del interés y el afecto que su entorno familiar le ha brindado, por otra parte, otros reciben constantemente mensajes negativos de parte de sus progenitores, debilitando y distorsionando sus autoconceptos. De tal manera, los niños entran en el medio escolar con un autoconcepto desarrollado, pero susceptible de ser modificado por el contorno familiar o por la interacción que tendrá durante los procesos de estudio.

\section{Depresión}

Alberdi, Taboada, Castro y Vásquez (2006), definen a la depresión como un síndrome o agrupación de síntomas, susceptibles de valoración y ordenamiento en unos criterios diagnósticos racionales y operativos. Por definición, el concepto de depresión recoge la presencia de síntomas afectivos, esfera de los sentimientos o emociones: tristeza patológica, decaimiento, irritabilidad, sensación subjetiva de malestar e impotencia frente a las exigencias de la vida, aunque, en mayor 
o menor grado, siempre están también presentes síntomas de tipo cognitivo, volitivo, o incluso somático. Se podría hablar de una afectación global de la vida pacífica, haciendo énfasis en la vida afectiva.

Del mismo modo, Piqueras, Martínez, Ramos, Rivero, García y Oblitas (2008) plantean la siguiente definición de depresión: "Es el resultado final de la interacción de múltiples factores constitucionales, evolutivos, ambientales e interpersonales que modifican las pautas de neurotransmisión entre hemisferios cerebrales y el sistema límbico". Por otra parte (Beck et al., 1983; Ellis, 1981; 1990; citado por Piqueras, Martínez, Ramos, Rivero, García y Oblitas, 2008), refieren que la depresión, clínicamente es un trastorno del estado de ánimo caracterizado por una sensación de una tristeza intensa superior a dos meses. Se produce por acontecimientos de la vida diaria, cambios químicos en el cerebro, efecto secundario de medicamentos, diversos trastornos físicos o médicos. Asimismo, Pardo, Sandoval y Umbarela, (2004; citado por Piqueras, Martínez, Ramos, Rivero, García y Oblitas, 2008) mencionan que es el punto más bajo de las fluctuaciones del estado de ánimo normal, de la vida emocional de todos los días; estado de infelicidad, tristeza o dolor emocional, que se presenta como reacción a una situación o evento displacentero, el cual es considerado como la causa de tal respuesta emocional.

Por su parte, Pereyra y Mussi (2005) mencionan que quizás la depresión provenga de una vida monótona, sin atractivo, uniformemente gris, en la cual cada día se deja caer en el alma una gota ínfima de desesperanza, por pequeña que sea, que va destruyendo imperceptiblemente el brillo de la existencia y extinguiendo al alegría, para finalmente, dejar traslucir ese fondo temido, sumiendo la existencia en una noche sin mañana.

Así también, la depresión es el estado que domina la existencia cuando dejamos de contemplar el cielo, perdemos la alegría de vivir y permitimos que los problemas se agolpen sobre nuestra cabeza hasta quitarnos el espíritu de lucha y llevarnos al derrotismo (Pereyra, 2006).

Finalmente, para Beck (2003), la define como una serie de concepciones negativas de sí mismo, del mundo y del futuro; mismas que conllevan a una serie de distorsiones sistemáticas en el procesamiento de la información. Entre tales series de distorsiones sistemáticas en el procesamiento se encuentra la sobre generalización, el pensamiento dicotómico, las inferencias arbitrarias, la abstracción selectiva y la personalización.

Por lo tanto se considera que la depresión y el autoconcepto están relacionados.

Revista Apunt. Univ. VOLUMEN VI • NÚMERO 2 p. 53 - 68 
Andrés Fernando Bartra Alegría - Epifanio Felimón, Guerra Sánchez -

Renzo Felipe Carranza Esteban

\section{Método}

\section{Diseño y tipo de investigación}

La investigación corresponde a un diseño no experimental transversal de tipo correlacional porque se buscó encontrar la relación entre autoconcepto y depresión en estudiantes universitarios de una Universidad Privada de Tarapoto (Hernández, Fernández y Baptista, 2010).

\section{Muestra}

Estuvo conformada por 465 estudiantes universitarios de una universidad privada de Tarapoto.

\section{Variables}

Autoconcepto y depresión

\section{Instrumentos}

Escala de Autoconcepto AF5 de García Musitu creada por Musitu, García y Gutiérrez, publicada en el año 1999, constituida por 30 ítems.

Inventario de Depresión de Beck Adaptado (BDI-IIA) por Carranza (2009), conformada por 21 ítems.

Para validar los instrumentos se solicitó el juicio de expertos, quienes evaluaron la coherencia, claridad, concisión, suficiencia, cumplimiento de objetivos, replicabilidad y relevancia de los ítems presentado en el instrumento; respecto a la justificación estadística se realizó una prueba piloto previa a la recolección de los datos con el fin de obtener información que ayude a mejorar la validez y confiabilidad del instrumento y detectar problemas en la validez de construcción y de contenido.

Se contó con la participación y colaboración voluntaria de los estudiantes de ambos sexos, entre edades que oscilan de 16 y 35 años. Una vez identificados los sujetos, se procedió a aplicar de manera individual y grupal el Cuestionario de Autoconcepto, (AF 5) y el Inventario de Depresión de Beck Adaptado (BDI-IIA).

Por otro lado, para el análisis de datos se utilizaron Técnicas Estadísticas Descriptivas y de correlación a un nivel de significancia $p<0.05$. Para procesar los datos y realizar los análisis correspondientes se utilizó el Software Estadístico para Windows SPSS. 


\section{Resultados}

\section{Propiedades Psicométricas de la Escala AF5 de Autoconcepto}

\section{Confiabilidad}

En cuanto a determinar la confiabilidad en su modalidad de consistencia interna se estimó el coeficiente Alfa de Cronbach, obteniéndose como resultado para la escala total un valor de 0.870 (Escala AF5 de Autoconcepto) siendo valorado como indicador de una elevada confiabilidad (Adkins, 1994), ver Tabla 1.

Tabla 1

Estimaciones de confiabilidad mediante el coeficiente de Alpha de la Escala AF5 de autoconcepto.

\begin{tabular}{ccc}
\hline Número de Ítem & Alfa de Cronbach & N \\
\hline 30 &, 870 & 465 \\
\hline
\end{tabular}

\section{Validez}

Como se puede apreciar en la Tabla 02, la prueba KMO y Prueba de Bartett, en conclusión, valores bajos del índice KMO desaconsejan la utilización de Análisis Factorial. En la tabla se observa que el índice KMO es de 0.934 siendo un valor meritorio para obtener el análisis factorial, lo que indica que el instrumento presenta validez.

Tabla 2

Estimaciones de validez mediante el coeficiente de KMO prueba Bartlett de la Escala AF5 de Autoconcepto.

\begin{tabular}{llr}
\hline Medida de adecuación muestral de Kaiser-Meyer-Olkin. &, 934 \\
Prueba de esfericidad de Bart- & Chi-cuadrado aproximado & 2651,825 \\
lett & Gl & 210 \\
& Sig. &, 000 \\
\hline
\end{tabular}

Propiedades Psicométricas del Inventario de Depresión de Beck Adaptado

\section{Confiabilidad}

Se aprecia en la tabla 03 el índice de Alpha $(0,888)$, indica que el instrumento es confiable

Revista Apunt. Univ. $\quad$ VOLUMEN VI • NÚMERO 2 p. 53 - 68 
Andrés Fernando Bartra Alegría - Epifanio Felimón, Guerra Sánchez Renzo Felipe Carranza Esteban

Tabla 3

Estimaciones de confiabilidad mediante el coeficiente de Cronbach del Inventario de Depresión de Beck Adaptado

\begin{tabular}{ccc}
\hline Número de Ítem & Alfa de Cronbach & N \\
\hline 21 &, 888 & 465 \\
\hline
\end{tabular}

Análisis descriptivo de la variable de autoconcepto global y sus dimensiones

La Tabla 04, nos indica que el $22,2 \%$ de los estudiantes universitarios presentan un nivel bajo en lo que respecta a su autoconcepto global; seguido por un $20,9 \%$ que se encuentra en el nivel muy bajo. Asimismo, un 19,1\% muestran tener autoconcepto muy alto, y con el mismo porcentaje de $18,9 \%$ los niveles de promedio y alto respectivamente.

Por otra parte, se aprecia, respecto al autoconcepto académico; que un $27,7 \%$ del total de la muestra se encuentra en un nivel promedio, mientras que el $25,8 \%$ se ubica en un nivel muy bajo, seguido por un $18,5 \%$ que posee un nivel bajo; además un 14,2\% obtienen un nivel alto, y finalmente un $13,8 \%$ obtiene un nivel muy alto.

Con respecto al autoconcepto social, se indica que un $27,1 \%$ presenta un nivel bajo, seguido por un $22,2 \%$ en un nivel muy bajo, por otro lado un $18,9 \%$ se ubica en un nivel promedio, $18,3 \%$ muestran tener un autoconcepto social alto y sólo un $13,5 \%$ presentan un nivel muy alto.

De otro lado, se muestra datos relativos a los niveles de autoconcepto emocional; se observa que la mayoría de participantes se ubica en un nivel muy bajo $(27,1 \%)$, seguido por un $20,6 \%$ en el nivel bajo y promedio, asimismo se observa que un $17,0 \%$ tiene un autoconcepto emocional muy alto y sólo un $14,6 \%$ se encuentra en el nivel alto.

Como se observa el $24,3 \%$ se encuentra en el nivel promedio, un $23,9 \%$ se ubica en el nivel muy bajo y bajo; mientras que un $14,6 \%$ en el nivel alto y solo un $13,3 \%$ en el nivel muy alto de Autoconcepto Familiar.

Con respecto al autoconcepto físico, el $25,4 \%$ se encuentra en nivel bajo, seguido por un $20,4 \%$ con un nivel muy bajo, mientras que el $19,1 \%$ se ubica en el nivel promedio, además un $17,8 \%$ corresponde al nivel alto y sólo el 17,2\% posee un nivel muy alto en el Autoconcepto Físico. 


\section{Tabla 4}

Análisis descriptivo de la variable de autoconcepto global y sus dimensiones

(Porcentaje entre paréntesis)

\begin{tabular}{|c|c|c|c|}
\hline $\begin{array}{l}\text { Dimensiones de } \\
\text { Autoconcepto }\end{array}$ & \multirow{2}{*}{$\begin{array}{l}\text { Categoría } \\
\text { Muy bajo }\end{array}$} & \multicolumn{2}{|c|}{ Frecuencia Porcentaje } \\
\hline & & 97 & $(20,9)$ \\
\hline & Bajo & 103 & $(22,2)$ \\
\hline \multirow[t]{5}{*}{ Global } & Promedio & 88 & $(18,9)$ \\
\hline & Alto & 88 & $(18,9)$ \\
\hline & Muy alto & 89 & $(19,0)$ \\
\hline & Muy bajo & 120 & $(25,8)$ \\
\hline & Bajo & 86 & $(18,5)$ \\
\hline \multirow[t]{5}{*}{ Académico } & Promedio & 129 & $(27,7)$ \\
\hline & Alto & 66 & $(14,2)$ \\
\hline & Muy alto & 64 & $(13,8)$ \\
\hline & Muy bajo & 103 & $(22,2)$ \\
\hline & Bajo & 126 & $(27,1)$ \\
\hline \multirow[t]{5}{*}{ Social } & Promedio & 88 & $(18,9)$ \\
\hline & Alto & 85 & $(18,3)$ \\
\hline & Muy alto & 63 & $(13,5)$ \\
\hline & Muy bajo & 126 & $(27,1)$ \\
\hline & Bajo & 96 & $(20,6)$ \\
\hline \multirow[t]{5}{*}{ Emocional } & Promedio & 96 & $(20,6)$ \\
\hline & Alto & 68 & $(14,6)$ \\
\hline & Muy alto & 79 & $(17,0)$ \\
\hline & Muy bajo & 111 & $(23,9)$ \\
\hline & Bajo & 111 & $(23,9)$ \\
\hline \multirow[t]{5}{*}{ Familiar } & Promedio & 113 & $(24,3)$ \\
\hline & Alto & 68 & $(14,6)$ \\
\hline & Muy alto & 62 & $(13,3)$ \\
\hline & Muy bajo & 95 & $(20,4)$ \\
\hline & Bajo & 118 & $(25,4)$ \\
\hline \multirow[t]{3}{*}{ Físico } & Promedio & 89 & $(19,1)$ \\
\hline & Alto & 83 & $(17,8)$ \\
\hline & Muy alto & 80 & $(17,2)$ \\
\hline Total & & 465 & $(100,0)$ \\
\hline
\end{tabular}

Revista Apunt. Univ.

VOLUMEN VI • NÚMERO 2 p. 53 - 68 
Andrés Fernando Bartra Alegría - Epifanio Felimón, Guerra Sánchez Renzo Felipe Carranza Esteban

Con respecto al autoconcepto global, en la Tabla 05, del total del sexo masculino, el $37,2 \%$ presentan un autoconcepto bajo y muy bajo, mientras que las mujeres el $47,7 \%$ se encuentran en el nivel bajo y muy bajo.

Con respecto al porcentaje que involucra las dimensiones del autoconcepto según género; el nivel muy bajo es significativo: dimensión académico $26,6 \%$, social $19,8 \%$, emocional $19,8 \%$, familiar $23,7 \%$ y físico $13,7 \%$ que corresponde al sexo masculino; así también, el sexo femenino presenta un nivel muy bajo de autoconcepto en las dimensiones: académico $25,8 \%$, social $22,2 \%$, emocional $32,9 \%$, familiar $24,0 \%$ y físico $26,4 \%$.

Tabla 5

Niveles de autoconcepto y sus dimensiones, según género (porcentaje entre paréntesis)

\begin{tabular}{|c|c|c|c|c|}
\hline \multirow{2}{*}{$\begin{array}{l}\text { Dimensiones de } \\
\text { Autoconcepto }\end{array}$} & \multirow[t]{2}{*}{ Categoría } & \multicolumn{2}{|c|}{ Sexo } & \multirow[t]{2}{*}{ Total } \\
\hline & & Masculino & Femenino & \\
\hline \multirow{5}{*}{ Global } & Muy bajo & $38(18,4)$ & $59(22,9)$ & $97(20,9)$ \\
\hline & Bajo & $39(18,8)$ & $64(24,8)$ & $103(22,2)$ \\
\hline & Promedio & $43(20,8)$ & $45(17,4)$ & $88(18,9)$ \\
\hline & Alto & $37(17,9)$ & $51(19,8)$ & $88(18,9)$ \\
\hline & Muy alto & $50(24,2)$ & $39(15,1)$ & $89(19,1)$ \\
\hline \multirow{5}{*}{ Académico } & Muy bajo & $55(26,6)$ & $65(25,2)$ & $120(25,8)$ \\
\hline & Bajo & $45(21,7)$ & $41(15,9)$ & $86(18,5)$ \\
\hline & Promedio & $54(26,1)$ & $75(29,1)$ & $129(27,7)$ \\
\hline & Alto & $25(12,1)$ & $41(15,9)$ & $66(14,2)$ \\
\hline & Muy alto & $28(13,5)$ & $36(14,0)$ & $64(13,8)$ \\
\hline \multirow{4}{*}{ Social } & $\begin{array}{l}\text { Muy bajo } \\
\text { Bajo }\end{array}$ & $\begin{array}{l}41(19,8) \\
60(29,0)\end{array}$ & $\begin{array}{l}62(24,0) \\
66(25,6)\end{array}$ & $\begin{array}{l}103(22,2) \\
126(12,7)\end{array}$ \\
\hline & Promedio & $40(19,3)$ & $48(18,6)$ & $88(18,9)$ \\
\hline & Alto & $42(20,3)$ & $43(16,7)$ & $85(18,3)$ \\
\hline & Muy alto & $24(11,6)$ & $39(15,1)$ & $63(13,5)$ \\
\hline \multirow{5}{*}{ Emocional } & Muy bajo & $41(19,8)$ & $85(32,9)$ & $126(27,1)$ \\
\hline & Bajo & $38(18,4)$ & $58(22,5)$ & $96(20,6)$ \\
\hline & Promedio & $45(21,7)$ & $51(19,8)$ & $96(20,6)$ \\
\hline & Alto & $37(17,9)$ & $31(12,0)$ & $68(14,6)$ \\
\hline & Muy alto & $46(22,2)$ & $33(12,8)$ & $79(17,0)$ \\
\hline \multirow{5}{*}{ Familiar } & Muy bajo & $49(23,7)$ & $62(24,0)$ & $111(23,9)$ \\
\hline & Bajo & $48(23,2)$ & $63(24,4)$ & $111(23,9)$ \\
\hline & Promedio & $50(24,2)$ & $63(24,4)$ & $113(24,3)$ \\
\hline & Alto & $28(13,5)$ & $40(15,5)$ & $68(14,6)$ \\
\hline & Muy alto & $32(15,5)$ & $30(11,6)$ & $62(13,3)$ \\
\hline
\end{tabular}




\begin{tabular}{lllll} 
& Muy bajo & $27(13,0)$ & $68(26,4)$ & $95(20,4)$ \\
Físico & Bajo & $48(23,2)$ & $70(27,1)$ & $118(2,4)$ \\
& Promedio & $41(19,8)$ & $48(18,6)$ & $89(19,1)$ \\
& Alto & $44(21,3)$ & $39(15,1)$ & $83(17,8)$ \\
& Muy alto & $47(22,7)$ & $33(12,8)$ & $80(17,2)$ \\
Total & & & \\
& & $207(44.5)$ & $258(55.5)$ & $465(100)$ \\
\hline
\end{tabular}

En la Tabla 06, el porcentaje más prevalente, entre los grupos etarios de acuerdo al autoconcepto global y sus dimensiones se ubican en el nivel muy bajo como se percibe a continuación: de 16-18 años un 30,3\% con respecto al autoconcepto emocional, de 19 a 21 años un $25,4 \%$ con respecto al autoconcepto académico y emocional, de 22 a 24 un 26,9\% con respecto al autoconcepto familiar, de 25 años a más el $34,8 \%$ con respecto al autoconcepto académico, y finalmente los estudiantes de 25 años a más un 30,4\% presentan un autoconcepto bajo con respecto al autoconcepto global.

Tabla 6

Niveles de autoconcepto y sus dimensiones, según las edades (porcentaje entre paréntesis)

\begin{tabular}{|c|c|c|c|c|c|c|}
\hline \multirow{2}{*}{$\begin{array}{l}\text { Autocon- } \\
\text { cepto }\end{array}$} & \multirow[t]{2}{*}{ Categorías } & \multicolumn{4}{|c|}{ Edades } & \multirow[t]{2}{*}{ Total } \\
\hline & & $16-18$ & $19-21$ & $22-24$ & 25 a más & \\
\hline \multirow{5}{*}{ Global } & Muy bajo & $44(19.3)$ & $39(23.1)$ & $9(20.0)$ & $5(21.7)$ & 97 (20.9) \\
\hline & Bajo & $60(26.3)$ & $30(17.8)$ & $6(13.3)$ & $7(30.4)$ & $103(22.2)$ \\
\hline & Promedio & $46(20.2)$ & $28(16.6)$ & $11(24.4)$ & $3(13.0)$ & 88 (18.9) \\
\hline & Alto & 39 (17.1) & $38(22.5)$ & $8(17.8)$ & $3(13.0)$ & 88 (18.9) \\
\hline & Muy alto & 39 (17.1) & $34(20.1)$ & $11(24.4)$ & $5(21.7)$ & 89 (19.1) \\
\hline \multirow{5}{*}{ Académico } & Muy bajo & $58(25.4)$ & $43(25.4)$ & $11(24.4)$ & $8(34.8)$ & $120(25.8)$ \\
\hline & Bajo & 45 (19.7) & 32 (18.9) & 7 (15.6) & $2(8.7)$ & 86 (18.5) \\
\hline & Promedio & 66 (28.9) & $46(27.2)$ & $10(22.2)$ & $7(30.4)$ & $129(27.7)$ \\
\hline & Alto & 31 (13.6) & $22(13.0)$ & $10(22.2)$ & $3(13.0)$ & 66 (14.2) \\
\hline & Muy alto & $28(12.3)$ & $26(15.4)$ & $7(15.6)$ & $3(13.0)$ & $64(13.8)$ \\
\hline \multirow{5}{*}{ Social } & Muy bajo & 45 (19.7) & 42 (24.9) & 12 (26.9) & $4(17.4)$ & $103(22.2)$ \\
\hline & Bajo & $67(29.4)$ & 37 (21.9) & $10(22.2)$ & $12(52.2)$ & $126(27.1)$ \\
\hline & Promedio & 45 (19.7) & 32 (18.9) & $8(17.8)$ & $3(13.0)$ & 88 (18.9) \\
\hline & Alto & $38(16.7)$ & 35 (20.7) & $9(20.0)$ & $3(13.0)$ & 85 (18.3) \\
\hline & Muy alto & $33(14.5)$ & 23 (13.6) & $6(13.3)$ & $1(4.3)$ & 63 (13.5) \\
\hline \multirow{5}{*}{ Emocional } & Muy bajo & $69(30.3)$ & $43(25.4)$ & $9(20.0)$ & $5(21.7)$ & $126(27.1)$ \\
\hline & Bajo & $51(22.4)$ & $29(17.8)$ & $8(17.8)$ & $8(34.8)$ & 96 (20.6) \\
\hline & Promedio & $46(20.2)$ & 36 (21.3) & $11(24.4)$ & $3(13.0)$ & 96 (20.6) \\
\hline & Alto & $30(13.2)$ & $28(16.6)$ & 7 (15.6) & $3(13.0)$ & $68(14.6)$ \\
\hline & Muy alto & $32(14.0)$ & 33 (19.5) & $10(22.2)$ & 4 (17.4) & 79 (17.0) \\
\hline
\end{tabular}

Revista Apunt. Univ. $\quad$ VOLUMEN VI • NÚMERO 2 p. 53 - 68 
Andrés Fernando Bartra Alegría - Epifanio Felimón, Guerra Sánchez Renzo Felipe Carranza Esteban

\begin{tabular}{clccccc} 
& Muy bajo & $55(24.1)$ & $39(23.1)$ & $12(26.7)$ & $5(21.7)$ & $111(23.9)$ \\
\multirow{5}{*}{ Familiar } & Bajo & $58(22.5)$ & $38(22.5)$ & $10(22.2)$ & $5(21.7)$ & $111(23.9)$ \\
& Promedio & $57(25.0)$ & $39(23.1)$ & $11(24.4)$ & $6(26.1)$ & $113(24.3)$ \\
& Alto & $29(12.7)$ & $30(17.8)$ & $6(13.3)$ & $3(13.0)$ & $68(14.6)$ \\
& Muy alto & $29(12.7)$ & $23(13.6)$ & $6(13.3)$ & $4(17.4)$ & $62(13.3)$ \\
& & & & & \\
\multirow{5}{*}{ Físico } & Muy bajo & $52(22.8)$ & $34(20.1)$ & $6(13.3)$ & $3(13.0)$ & $95(20.4)$ \\
& Bajo & $55(24.1)$ & $48(28.4)$ & $7(15.6)$ & $8(34.8)$ & $118(25.4)$ \\
& Promedio & $51(22.4)$ & $25(14.8)$ & $10(22.2)$ & $3(13.0)$ & $89(19.1)$ \\
& Alto & $32(14.0)$ & $31(18.3)$ & $14(31.1)$ & $6(26.1)$ & $83(17.8)$ \\
& Muy alto & $38(16.7)$ & $31(18.3)$ & $8(17.8)$ & $3(13.0)$ & $80(17.2)$ \\
Total & & & & & & \\
\hline
\end{tabular}

En cuanto a la escala de depresión en los estudiantes universitarios, se observa en la Tabla 07, que el $26,2 \%$ se ubica en el nivel moderado, seguido por una diferencia mínima de $0,3 \%(26,2 \%)$ quienes poseen un nivel mínimo de depresión, mientras que un $23,9 \%$ se ubica en el nivel leve, y finalmente un 23,4\% presenta un nivel severo de depresión.

Tabla 7

Niveles de Depresión (Porcentaje entre paréntesis)

\begin{tabular}{|c|c|c|}
\hline Nivel de Depresión & Frecuencia & Porcentaje \\
\hline Mínimo & 122 & $(26,2)$ \\
\hline Leve & 111 & $(23,9)$ \\
\hline Moderado & 123 & $(26,5)$ \\
\hline Severo & 109 & $(23,4)$ \\
\hline Total & 465 & $(100,0)$ \\
\hline
\end{tabular}

Se percibe en la Tabla 08, que un $49 \%$ de estudiantes universitarios se ubican entre las edades de 16 y 18 años de edad y quienes presentan mayores niveles de depresión.

Tabla 8

Niveles de depresión según sexo y edad (porcentaje entre paréntesis)

\begin{tabular}{|c|c|c|c|c|c|}
\hline \multirow{2}{*}{ Características } & \multicolumn{4}{|c|}{ Niveles de depresión } & \multirow[b]{2}{*}{ Total } \\
\hline & Mínimo & Leve & Moderado & Severo & \\
\hline \multicolumn{6}{|l|}{ Sexo } \\
\hline Masculino & $59(48,4)$ & $48(43,2)$ & $54(43,9)$ & $46(42,2)$ & $207(44,5)$ \\
\hline Femenino & $63(52,6)$ & $63(56,8)$ & $69(56,1)$ & $63(57,8)$ & $258(55,5)$ \\
\hline
\end{tabular}




Edad
\begin{tabular}{rccccc} 
16-18 años & $57(46,7)$ & $51(45,9)$ & $66(53,7)$ & $54(49,5)$ & $228(49,0)$ \\
$19-21$ años & $37(30,3)$ & $47(42,3)$ & $38(30,9)$ & $47(43,1)$ & $169(36,3)$ \\
$22-24$ años & $19(15,6)$ & $9(8,1)$ & $14(11,4)$ & $3(2,8)$ & $45(9,7)$ \\
25 a más & $9(7,4)$ & $4(3,6)$ & $5(4,6)$ & $5(4,1)$ & $23(4,9)$ \\
& & & & & \\
Total & $\mathbf{1 2 2 ( 2 6 . 2 )}$ & $\mathbf{1 1 1 ( 2 3 , 9 )}$ & $\mathbf{1 2 3 ( 2 6 , 5 )}$ & $\mathbf{1 0 9 ( 2 3 , 4 )}$ & $\mathbf{4 6 5 ( 1 0 0 , 0 )}$ \\
\hline
\end{tabular}

\section{Relación entre autoconcepto y depresión}

El coeficiente de correlación de Pearson, indica que existe una relación inversa y altamente significativa entre autoconcepto y depresión ( $r=-, 556$; $\mathrm{p}<0.01$ ). Los valores mostrados indican que a menor autoconcepto mayor depresión (Tabla 09).

Tabla 9

Análisis de correlación entre autoconcepto y depresión

\begin{tabular}{lcc}
\hline & \multicolumn{2}{c}{ Depresión } \\
\cline { 2 - 3 } Autoconcepto & $r$ & $\mathrm{P}$ \\
&,$- 462 * *$ &, 000 \\
\hline
\end{tabular}

**. La correlación es significativa al nivel 0,01 (bilateral).

\section{Relación entre las dimensiones de autoconcepto y depresión}

En la Tabla 10 se observa que existe una correlación inversa y altamente significativa entre las dimensiones del autoconcepto y depresión; académico $\left(r=-, 317^{* *} ; p=0,00\right)$, social $\left(r=-, 304^{* *} ; p=0,00\right)$, emocional $\left(r=-, 376^{* *} ; p=\right.$ $0,00)$,familiar $\left(r=-, 266^{* *} ; p=0,00\right)$ y físico $(r=-, 340 * * ; p=0,00)$, quiere decir que cuanto menor sea el autoconcepto académico, social, emocional, familiar y físico; mayor será la depresión. 
Andrés Fernando Bartra Alegría - Epifanio Felimón, Guerra Sánchez Renzo Felipe Carranza Esteban

Tabla 10

Correlación entre las dimensiones del autoconcepto y depresión:

\begin{tabular}{lcc}
\hline Dimensiones de autoconcepto & \multicolumn{2}{c}{ Depresión } \\
\hline & $\mathrm{r}$ & $\mathrm{p}$ \\
A_Académico &,$- 317^{* *}$ &, 000 \\
A_Social &,$- 304^{* *}$ &, 000 \\
A_Emocional &,$- 376^{* *}$ &, 000 \\
A_Familiar &,$- 266^{* *}$ &, 000 \\
A_Físico &,$- 340^{* *}$ &, 000 \\
\hline
\end{tabular}

*. La correlación es significativa al nivel 0,01 (bilateral).

\section{Discusión y conclusión}

Una de las contribuciones de mucha importancia del presente estudio es la confirmación de la validez y confiabilidad de los instrumentos empleados. Asimismo, es meritorio resaltar que en la presente investigación los estudiantes universitarios de una universidad privada de Tarapoto muestran niveles de autoconcepto general que fluctúan entre muy bajo y promedio $(62,0 \%)$. En contraste con lo mencionado, Reyes (2003), en un estudio desarrollado en estudiantes de Psicología, de la Universidad Nacional Mayor de San Marcos, encontró que el $88,7 \%$ de ellos muestra un autoconcepto global entre niveles promedio y alto; comparando estos hallazgos con otros estudios efectuados en distintos lugares del mundo, los indicadores encontrados son superiores; esto se explica considerando que para medir el autoconcepto en estudiantes universitarios, se utilizaron diversos instrumentos y en realidades diferentes; es así, que en un estudio realizado por Schmidt (2010) en estudiantes de la Universidad Católica de Argentina, se encontró niveles de autoconcepto que interfieren en la motivación para que los estudiantes continúen el curso de sus carreras; asimismo, cabe hacer mención que son las mujeres quienes presentan mayores dificultades en sus niveles de autoconcepto.

Por otro lado, la prevalencia de depresión en los estudiantes universitarios que forman parte de este estudio, es un tema de investigación que suscita el interés de estudiosos, que son conscientes de la influencia de la enfermedad, en la calidad de respuesta de los estudiantes frente a su rendimiento académico, relaciones interpersonales, calidad de trabajo, en general en sus quehaceres cotidianos. 
Al haberse realizado el análisis descriptivo de la variable depresión se halló que el $23.9 \% \%$ presentan un nivel leve de depresión, datos similares se aprecian en el estudio de Sáenz (2011) quien encontraron que el $22 \%$ de los estudiantes de una preparatoria de Chihuana presentaron depresión leve siendo un foco de atención para atender el área emocional de los estudiantes. Asitambien en el estudio realizado por Carranza (2012) se observa que el 53\% de estudiantes universitarios de lima metropolitana presentan niveles de depresión.

Otro hallazgo importante en el estudio es que los estudiantes que se ubican entre las edades de 16 a 21 años de edad son lo que presentan mayores niveles de depresión, datos semejantes se aprecian en la investigación llevaba a cabo por Carranza (2012). Es posible que esto se deba a las exigencias académicas a que son sometidos los estudiantes universitarios como también en este proceso de transición de colegio a universidad suelen haber desequilibrios emocionales.

De otro lado, un estudio realizado por Gutiérrez, Montoya, Toro, Briñón, Rosa y Salazar (2010), en una muestra de 1344 estudiantes universitarios, comprendidos entre 16 y 37 años de edad, de ambos sexos, demostraron una relación entre la severidad de la depresión y las dificultades de tipo académico moderado y severo: siendo las mujeres más susceptibles de deprimirse; datos semejantes de aprecian en los resultados obtenidos siendo que el 55,5\% de mujeres presentan niveles de depresión.

Asimismo en el análisis de correlación entre cada dimensión del autoconcepto, con la depresión, se muestran los siguientes resultados: académico $\left(r=-, 317^{* *} ; p=0,00\right)$, social $\left(r=-, 304^{* *} ; p=0,00\right)$, emocional $\left(r=-, 376^{* *} ; p=\right.$ $0,00)$,familiar $\left(r=-, 266^{* *} ; p=0,00\right)$ y físico $\left(r=-, 340^{* *} ; p=0,00\right)$; se destaca que existe correlación inversa altamente significativa, lo que indica que los niveles actuales de autoconcepto en sus diversas dimensiones, de los participantes en nuestro estudio, inciden sobre los niveles de depresión que experimentan.

Ante estos resultados se debe tomar en consideración al autoconcepto como factor principal para lograr un nivel adecuado en el área emocional, fortalecer el autoconcepto es importante puesto que influye en la formación y el desarrollo logrando construir la confianza en sí mismo para enfrentar obstáculos, dedicando mayor esfuerzo para alcanzar metas trazadas.

Renzo Felipe Carranza Esteban

Universidad Peruana Unión, Perú email: renzo.carranza@upeu.edu.pe

Recibido: 24 de noviembre de 2015 Aceptado: 22 de enero de 2016

Revista Apunt. Univ. VOLUMEN VI • NÚMERO 2 p. 53 - 68 
Andrés Fernando Bartra Alegría - Epifanio Felimón, Guerra Sánchez Renzo Felipe Carranza Esteban

\section{Referencias}

Alberdi, J., Taboada, O., Castro, C., y Vásquez, C. (2006). Depresión. Guías Clínicas, 6 (11), 1-6. Recuperado de http://www.scamfyc.org/documentos/Depresion\%20Fisterra. pdfÁlvarez, Ramírez, Silva, Coffin y Jiménez (2009)

Alfaro, R. (2009). Autococnepto y Conducta disciplinaria de los alumnos del nivel secundario de la Institución Educativa Adventista Miraflores. (Tesis inédita de maestría). Universidad Peruana Unión, Lima.

Beck, A. (2003). Depresión aspectos psicológicos. Océano S.A.

Carranza, R. (2012). Depresión y características demográficas asociados en estudiantes lideres universitarios de lima metropolitana. Revista Apuntes Universitarios, 2, 79-90. Recuperado de http://apuntesuniversitarios.upeu.edu.pe/index.php/revapuntes/article/view/24/22

Esnaola, I. (2008). El autoconcepto físico durante el ciclo de vital. Anales de psicología, 24(1), 1-8. Recuperado de http://www.um.es/analesps/v24/v24_1/01-24_1.pdf

García F., y Musitu, G. (2001). Manual AF5 Autoconcepto. Madrid: TEA Ediciones.

García, J., Musitu, G., Riquelme, E., y Riquelme, P. (2011). A Confirmatory Factor Analysis of the "Autoconcepto Forma 5" Questionnaire in Young Adults from Spain and Chile. The Spanish Journal of Psychology, 14 (2), 648-658. Recueperado de http://www.ncbi.nlm. nih.gov/pubmed/22059311

Goñi, A., Esnaola, I., Ruiz de Azua, A., Rodriguez, A., y Zulaika, L. (2003). Autoconcepto físico y desarrollo personal: perspectivas de investigación. Revista de Psicodidáctica, 15(16), 7-62. Recuperado de http://redalyc.uaemex.mx/redalyc/pdf/175/17515081003.pdf

González, M., y Tourón, J. (1994). Autoconocimiento y rendimiento escolar. Susimplicaciones en la motivación y en la autorregulación del aprendizaje. Barañain: Eunsa.

Gutiérrez, J., Montoya, L., Toro, B., Briñón, M., Rosas, E. Y Salazar, L. (2010). Depresión en estdudiantes universitarios y su asociación con el estrés académico. Revista CES Medicina, 24 (1), 7-17. Recuperado de http://redalyc.uaemex.mx/redalyc/pdf/2611/261119491001.pdf

Hernández, R., Fernández, C., y Baptista, P. (2010). Metodología de la Investigación. México: Mc GrawHill.

Musitu, G., García, F., y Gutiérrez, M. (1999). Autoconcepto Forma 5 (AFA). Madrid: TEA, Ediciones, S.A.

Pereyra, M. (2006). Psicología Positiva. Lima: Ed. Imprenta unión.

Pereyra, M., y Mussi, C. (2005). Sea feliz, ¿cómo vencer la depresión y controlar la ansiedad? Montemorelos: Montemorelos México Ed.

Piqueras, J., Martínez, A., Ramos, V., Rivero, R., García, L., y Oblitas, L. (2008). Ansiedad, depresión y salud. Suma Psicológica, 15 (1), 43-73. Recuperado de http://redalyc.uaemex.mx/ pdf/1342/134212604002.pdf

Schmidt, A. (2010). "Autoconcepto y motivaciones vocacionales en estudiantes de primer año de carreras de Humanidades y Ciencias Exactas" (Tesis para obtener título de Psicóloga, Pontificia Universidad Católica de Argentina). Recuperado de http://bibliotecadigital.uca. edu.ar/repositorio/tesis/autoconcepto-motivaciones-vocacionales-en-estudiantes.pdf

Vega, M., y Capa, W. (2009). Influencia del autoconcepto, las estrategias de aprendizaje y percepción acerca del docente en el rendimiento académico de los alumnos de Psicología, Año 2007. (2009). Revista de Investigación Universitaria 1(1), 105-114. 